\title{
Resolving Response, Decision, and Strategic Control: Evidence for a Functional Topography in Dorsomedial Prefrontal Cortex
}

\author{
Vinod Venkatraman, ${ }^{1,2,3}$ Alexandra G. Rosati, ${ }^{2}$ Adrienne A. Taren, ${ }^{2}$ and Scott A. Huettel ${ }^{1,2,3}$ \\ ${ }^{1}$ Brain Imaging and Analysis Center, Durham, North Carolina 27710, and ${ }^{2}$ Center for Cognitive Neuroscience and ${ }^{3}$ Department of Psychology and \\ Neuroscience, Duke University, Durham, North Carolina 27710
}

The dorsomedial prefrontal cortex (DMPFC) plays a central role in aspects of cognitive control and decision making. Here, we provide evidence for an anterior-to-posterior topography within the DMPFC using tasks that evoke three distinct forms of control demandsresponse, decision, and strategic - each of which could be mapped onto independent behavioral data. Specifically, we identify three spatially distinct regions within the DMPFC: a posterior region associated with control demands evoked by multiple incompatible responses, a middle region associated with control demands evoked by the relative desirability of decision options, and an anterior region that predicts control demands related to deviations from an individual's preferred decision-making strategy. These results provide new insight into the functional organization of DMPFC and suggest how recent controversies about its role in complex decision making and response mapping can be reconciled.

\section{Introduction}

The medial prefrontal cortex contributes to a variety of cognitive functions like conflict monitoring (Botvinick et al., 2001; Pochon et al., 2008), error detection (Carter et al., 1998), outcome evaluation (Bush et al., 2002; Gehring and Willoughby, 2002), reinforcement learning (Kennerley et al., 2006), decision making under uncertainty (Rushworth et al., 2005; Rushworth and Behrens, 2008), emotions (Etkin et al., 2006), and social interactions (Amodio and Frith, 2006; Behrens et al., 2008, 2009). Meta-analyses indicate that these functions are supported by brain regions along the anterior cingulate sulcus and extending dorsally, hereafter collectively referred to as dorsomedial prefrontal cortex (DMPFC) (Ridderinkhof et al., 2004; Beckmann et al., 2009).

Several lines of evidence suggest that DMPFC may possess a topographic organization of function. Studies of the anterior cingulate cortex, typically defined to encompass medial prefrontal cortex more broadly and ventrally than DMPFC, have found distinctions based on emotional content (Bush et al., 2000) and social relevance (Behrens et al., 2008) of task stimuli. The DMPFC has also been postulated to shape activation in dorsolateral PFC (DLPFC) (Kerns et al., 2004; Mériau et al., 2006), and its subregions differ in their anatomical connectivity accordingly

Received June 9, 2009; revised Aug. 2, 2009; accepted Sept. 15, 2009.

This research was supported by the National Institute of Mental Health (Grant 70685) and by the National Institute of Neurological Disease and Stroke (Grant 41328). S.A.H. was supported by an Incubator Award from the Duke Institute for Brain Sciences. We thank James Bettman, John Payne, and Mary Frances Luce for discussions about the attribute-balancing paradigm. V.V., A.G.R., and S.A.H. designed the experiments. V.V. and A.G.R. led the programming of the experiments, data collection, and data analysis, in collaboration with S.A.H. A.A.T. assisted with general data analysis and led the meta-analysis. All authors contributed to writing and editing the final manuscript.

Correspondence should be addressed to Scott A. Huettel, Center for Cognitive Neuroscience, Box 90999, Duke University, Durham, NC 27710. E-mail: scott.huettel@duke.edu.

D01:10.1523/JNEUROSCI.2708-09.2009

Copyright $\odot 2009$ Society for Neuroscience ～0270-6474/09/2913158-07\$15.00/0
(Beckmann et al., 2009). Given evidence that the DLPFC is organized along a posterior-to-anterior gradient of increasing abstraction (Koechlin et al., 2000, 2003; Christoff et al., 2003), we hypothesized that a similar organization would exist within DMPFC. Specifically, we predicted that posterior DMPFC, which shows greater connectivity to motor and premotor regions, would support response-related control, while more anterior regions, which show greater connectivity to DLPFC, would support decision-related control. Moreover, based on evidence that DMPFC is also recruited for choices that run counter to behavioral strategies (Paulus et al., 2002; De Martino et al., 2006; Hampton and O'Doherty, 2007; Venkatraman et al., 2009), we also predicted that strategy-related control would evoke activation in the most anterior aspect of DMPFC.

We evoked these three distinct forms of control-response, decision, and strategy-within the same subjects and with distinct behavioral covariates. To evoke response-related control demands, we used a counting Stroop task (see Fig. $1 A$ ), using the subject-by-subject response-incongruency effect as a measure of response-related control (see Fig. $2 A$ ). For decision and strategic control demands, we used a decision-making task (see Fig. $1 B$ ), where subjects had to decide between two stocks based on their attributes: the balanced stock had equal ratings on two attributes, while the extreme stock had a high rating on one attribute but a lower rating on the other. Subjects showed a strong bias for an attribute-balancing strategy, consistent with previous studies (Chernev, 2004, 2005). On each trial, the decision-related control demand was characterized by the difference in relative desirability of the two stocks (see Fig. 2 B). Strategic control demands were characterized according to the degree of bias toward the balancing strategy within each individual (see Fig. 2C). Should these three measures evoke distinct foci of activation, as predicted, 
A
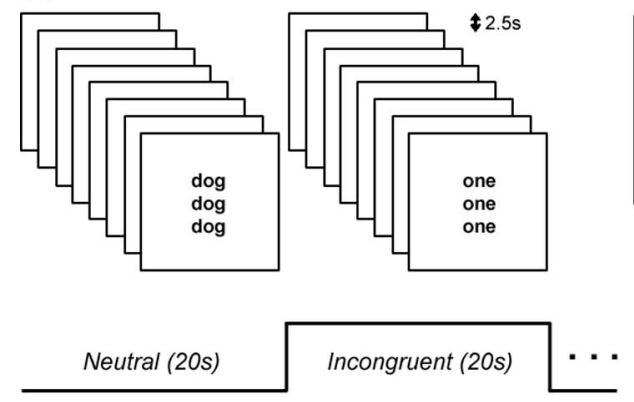

Counting Stroop Task
B

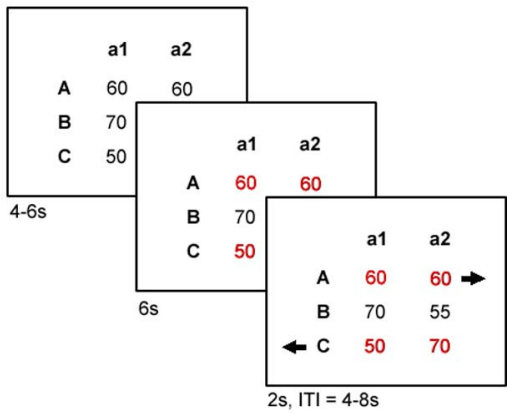

Attribute Balancing Task

Figure 1. Schematic of experimental task and behavioral results. $A$, Each participant completed one run of the counting Stroop task. We used a block design with alternating blocks of neutral and incongruent trials. Each trial lasted $2.5 \mathrm{~s}$, and subjects were instructed to respond as quickly and accurately as possible. Each block consisted of 8 trials and lasted 20 s. B, Subjects were first shown, for 4-6s, three anonymized stocks with ratings on two attributes. Then, two stocks were highlighted in red, whereupon subjects had $6 \mathrm{~s}$ to decide which they preferred. Finally, after two arrows identified the buttons corresponding to the potential choices, subjects indicated their choice by pressing the corresponding button as soon as possible. Here, stock A represents a balanced option (with equal ratings on both attributes), while stock C represents an extreme option (with good rating on attribute a2 but poor rating on a1). In this example, both highlighted stocks have similar expected value, but the stocks differed in expected value on other trials. The next trial appeared after a variable interval of 4,6, or $8 \mathrm{~s}$.

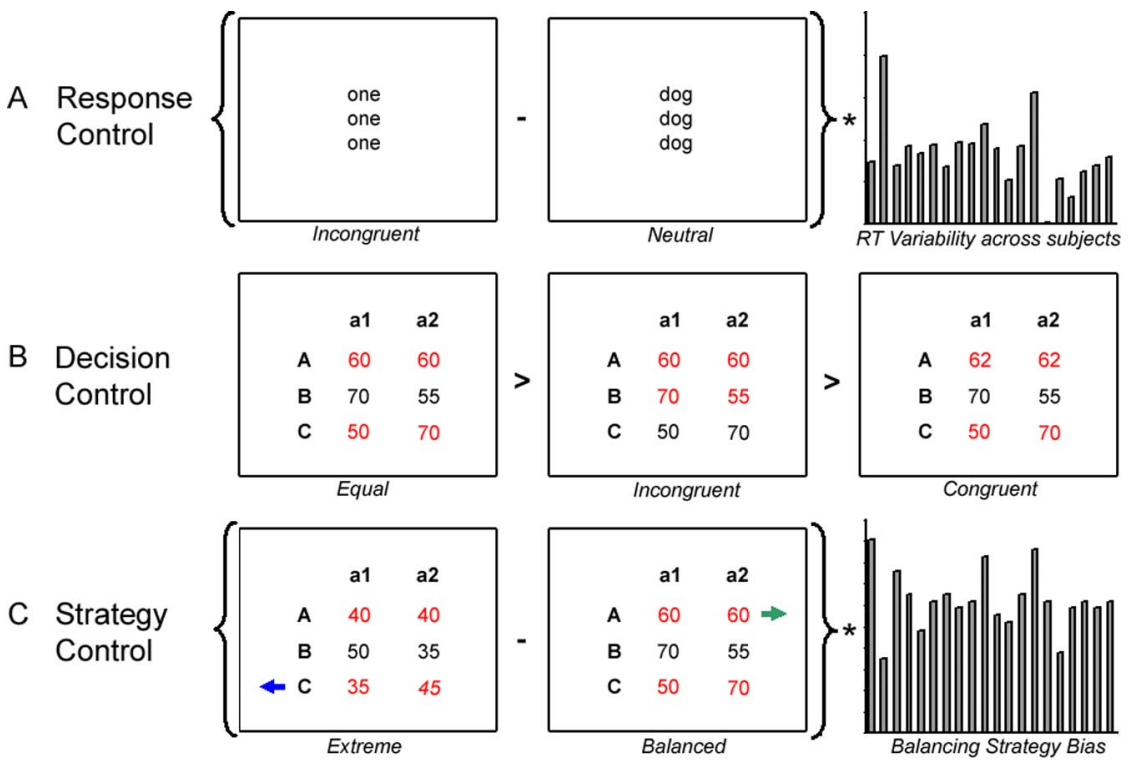

Figure 2. Response-, decision-, and strategy-related control. $\boldsymbol{A}$, The degree of response-related control was defined by the contrast between incongruent and neutral trials in the Stroop task, covaried across subjects by the relative response time difference between those two trial types. $\boldsymbol{B}$, The degree of decision-related control was defined according to the similarity in the desirability of the decision options in our attribute-balancing task. Maximal control demands occurred in the equal expected-value trials, intermediate demands in the incongruent trials, and minimal control demands in the congruent trials. C, Strategy-related control demands were defined by taking the difference in activation between extreme and balanced choices and covarying that contrast across subjects with degree of bias toward the balanced choice option. Maximal strategy-related control demands were present when a subject with a strong bias toward one option (either extreme or balanced) chose the other option, while minimal control demands were present when subjects made choices consistent with their usual bias.

there would be strong evidence in support of a functional topography within DMPFC.

\section{Materials and Methods}

Subjects. Twenty-three young adults $(7$ males, mean age $=21.9)$ participated in this study. All subjects acclimated to the scanning environment using a mock MRI scanner and participated in two short practice runs consisting of six trials each, one inside and one outside of the MRI scan-

ner. Three subjects were excluded before data analysis - two due to excessive motion and one for poor behavioral performance-leaving a total of 20 subjects in the final analyses. We used an incentive-compatible design in which subjects received monetary compensation both for participation and for their performance in the experiment (see supplemental materials for details, available at www.jneurosci.org). All subjects gave written informed consent as part of protocols approved by the Institutional Review Boards of Duke University and Duke University Medical Center.

Stimuli and experimental conditions. Responserelated control demands were evoked using a counting Stroop task (Bush et al., 1998). We used a block design for this task (Fig. 1A). In the neutral condition, subjects were presented with multiple repetitions of animal words (e.g., dog, cat) and asked to count the number of times the word was presented. In the incongruent condition, they were presented with multiple repetitions of number words (one, two, three, or four) and asked to count the number of times the word was presented. However, the number of repetitions was always incongruent with the number word itself (for example, "two" presented four times). Each word was repeated between 1 and 4 times, and subjects responded by pressing the corresponding button on a 4 button response box. We calculated, for each subject, the magnitude of the Stroop incongruency effect, measured as the differences in response times between the incongruent and neutral conditions and used it as an index of response-control demands (Fig. 2A).

Decision-related and strategy-related control demands were evoked using an attributebalancing task where all subjects were presented with a total of 90 sets of three real stocks, each rated on two attributes that provided real metrics of stock performance (Fig. $1 B$ ). To minimize the influence of subjects' previous knowledge or financial biases, the identity of the stocks and the nature of the attributes were concealed from the subjects until after the experiment. On each trial, the three stocks were labeled as "A," "B," and "C," and the two attributes were labeled as "al" and "a2" and shown as percentile rankings. The subjects' task was to predict the better-performing stock based only on these attributes.

All choice trials involved one stock that had balanced values for both attributes, one stock that had a good rating on attribute al but a poorer rating on attribute $\mathrm{a} 2$, and a third stock in which this pattern was reversed with slightly varying attribute ratings (see supplemental materials for more details, available at www.jneurosci. org). In each trial, subjects had to choose between a balanced stock and an extreme stock that was randomly selected from the other two alternatives for that trial. Choosing the balanced stock was consistent with the simple attribute-balancing heuristic identified in previous studies using similar paradigms (Chernev, 2004, 2005).

In addition, we randomly interspersed six catch trials, where the extreme alternative had higher ratings than the balanced option for both attributes and thus was the more desirable option. These trials thus served to ensure that subjects followed instructions and attended to the task. One subject who performed contrary to expectations in the catch trials was excluded from further analysis. 
We manipulated the decision-related control demands across trials by varying the expected-value relationship (defined as the sum of two attributes for each stock) between the balanced and extreme choices (Fig. $2 B)$. In 36 trials (congruent condition), the balanced stock had a higher expected value compared to the alternative option. In another 36 trials (incongruent condition), the balanced stock had a lower expected value compared to the alternative option. In the remaining 18 choice trials (equal condition), the expected value for the balanced stock was equal to the expected value of the alternative option. Decision-related control demands were minimal (i.e., where decisions were easiest) in the congruent condition, followed by the incongruent condition, and were maximal in the equal condition (i.e., where both choices have similar expected value).

Finally, we defined strategy-related control demands (Fig. 2C) according to the degree to which a given choice (i.e., balanced or extreme, on each trial) deviates from the individual's empirical strategy preferences (i.e., the proportion of balanced choices across all trials). Subjects who were normally biased toward the balanced option would exhibit maximal strategy-related control when they choose the extreme option. However, subjects who were biased toward the extreme option would exhibit maximal strategy-related control when they choose the balanced option. This analysis approach counterbalances for any main effects of either choice type, ensuring that our effects indeed reflect strategy-related control.

Experimental design and task timing. Subjects first participated in four runs of the attribute-balancing decision task, each containing 24 gambles and lasting $\sim 6 \mathrm{~min}$. Each trial began with the display of all three stocks for 4 or $6 \mathrm{~s}$. Subjects were instructed previously to examine the stocks as presented (Fig. $1 B$ ). Subsequently, two of the three stocks were highlighted in red (one involving balanced attributes and other extreme attributes). Subjects were instructed to choose between one of these two stocks and incentivized with a monetary bonus for picking the betterperforming stock (see supplemental materials, available at www. jneurosci.org). They had $6 \mathrm{~s}$ to make their choice, whereupon two arrows appeared specifying which button corresponded to which choice. The association of the buttons to choice was random, with equal probabilities of each option being associated with left or right buttons. The mapping of buttons for each trial was not revealed until the response phase, further reducing any possibilities of response-related incongruency effects during the decision phase. Subjects were instructed to arrive at their decision during the $6 \mathrm{~s}$ interval and to press the button corresponding to their choice as soon as the arrows appeared. Response times were defined as the interval between the appearance of arrows and the button-press response. The decision and response phases were explicitly separated to prevent the contamination of decision effects with response-preparation effects. During the intertrial interval of $4-8 \mathrm{~s}$, a fixation cross was displayed on the screen. Notice that no feedback was provided at the end of each trial, and hence, there was no explicit learning during the decision phase of the task.

After four runs of the decision task, all subjects completed one run of the counting Stroop task. To maximize power for the contrast between neutral and incongruent trials, our design alternated between blocks of six trials of each type ( $3 \mathrm{~s}$ between trial onsets, $18 \mathrm{~s}$ blocks, 17 blocks per run), without any fixation or nontask blocks. A final 6 min run, whose data are not discussed in this manuscript, resolved a subset of the trials from the decision phase to actual stock labels and attributes. The winning stock was then identified based on data we collected on the real performance of these stocks (see supplemental materials, available at www. jneurosci.org), and subjects received a monetary bonus based on the performance of their selected stock on two randomly chosen trials.

Before the fMRI data collection, subjects had the opportunity to practice the experimental task (without reward) in two six-trial blocks, one presented outside the MRI scanner and the other presented within the MRI scanner but before collection of the fMRI data. All stimuli were created using the Psychophysics Toolbox for MATLAB (Brainard, 1997; Pelli, 1997) and were presented to the subjects via MR-compatible liquid crystal display goggles. Subjects responded with the index fingers of each hand via an MR-compatible response box.

Imaging methods. We acquired fMRI data on a 4T GE scanner using an inverse-spiral pulse sequence with the following parameters: repetition time $=2000 \mathrm{~ms}$; echo time $=30 \mathrm{~ms} ; 34$ axial slices parallel to the AC-PC plane, with voxel size of $3.75^{\star} 3.75^{\star} 3.8 \mathrm{~mm}$. High-resolution threedimensional full-brain anatomical images with a spoiled gradient recalled sequence were acquired and used for normalizing and coregistering individual subjects' data.

Analysis was performed using FEAT (FMRI Expert Analysis Tool) Version 5.63, part of FSL (FMRIB's Software Library; www.fmrib.ox.ac. $\mathrm{uk} / \mathrm{fsl}$ ) package (Smith et al., 2004). We removed artifacts in the fMRI timeseries using MELODIC and in-house scripts, with three a priori criteria for identifying noise-related components: power at high frequencies, rapid changes within short time windows, and correlation with estimated head motion. The following prestatistics processing steps were applied: motion correction using MCFLIRT (Motion Correction using FMRIB's Linear Registration Tool), slice-timing correction, removal of nonbrain voxels using BET (Brain Extraction Tool), spatial smoothing with a Gaussian kernel of full-width at half-maximum $8 \mathrm{~mm}$, and highpass temporal filtering. Registration to high resolution and standard images was performed using FLIRT (FMRIB's Linear Registration Tool).

General linear model analyses. For the response-related control analysis, we used one regressor to model activation in the incongruent blocks of the Stroop task (i.e., treating the neutral blocks as baseline). We introduced the mean response-time difference between incongruent and neutral trials for each subject as a covariate in the across-subjects regression analysis to determine the focus of activation for response-related control. The decision-related control analysis used six total regressors to model activation in the attribute-balancing task: three task regressors for the three conditions (congruent, incongruent, and equal), one regressor for the initial presentation of the stocks, one regressor for catch trials, and one regressor for the response period (scaled by response time). We defined active voxels using a conjunction analysis of [equal $>$ incongruent] and [incongruent $>$ congruent]. The model used for the strategyrelated control analysis contained five total regressors: two regressors modeling the balanced and extreme choices of subjects in conditions that cause the greatest decision conflict (trials where the absolute magnitude of expected value difference between the two options was $<8$ ), one regressor modeling the responses in the remaining conditions, one regressor for the initial presentation of the stocks, and one regressor to model the subject responses. We introduced each subject's relative bias toward the balancing strategy ( $z$-transformed proportion of balancing choices) as a covariate in the contrast between the balanced and extreme choice regressors.

Event-related regressors were generated by convolving impulses at the onsets of events of interest with a double-gamma hemodynamic response function. Within-subject analyses were performed using a fixed-effects model across runs for each subject. Across-subjects analyses were performed using FLAME (FMRIB's Local Analysis of Mixed Effects) Stage 1, which is FSL's implementation of mixed-effect analyses. All statistical images were thresholded using clusters determined by $z>2.3$ and a whole-brain-corrected cluster-significance threshold of $p<0.05$. The same analysis procedures and thresholding were used for analyses involving a between-subjects covariate. The MRIcron package was used for visualizing brain images (Rorden et al., 2007).

\section{Results}

\section{Behavior}

For the Stroop task, subjects were significantly more accurate in the neutral compared to the incongruent condition $\left(t_{(19)}=\right.$ 2.46, $p<0.05)$. Similarly, subjects also took significantly longer in the incongruent condition compared to the neutral condition $\left(t_{(19)}=3.88, p<0.001\right)$. Together, these findings are consistent with increased demands for response-related control in the incongruent condition.

For the attribute-balancing task, subjects showed a significant bias toward the balanced alternative, preferring it in $58 \%$ of the trials. Within the equal condition, they preferred the balanced alternative in $65 \%$ of the trials $\left(t_{(19)}=3.9, p<0.005\right)$. Despite this overall bias, subjects' choices were still sensitive to changes in expected value. Consistent with our predictions for decision- 


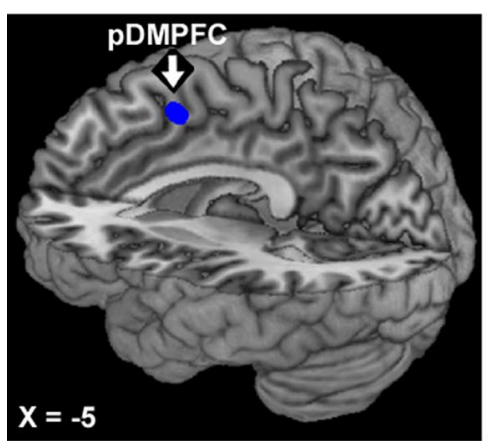

Response Control

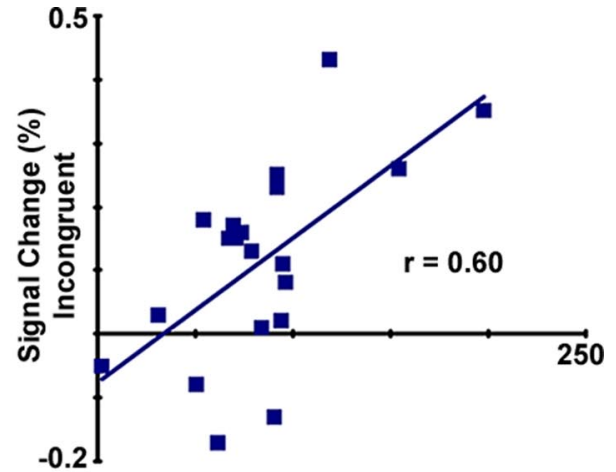

RT Variability (ms)

Figure 3. $\mathrm{DDMPFC}$ activation predicts response-related control demands. In the counting Stroop task, activation for incongruent trials in the posterior DMPFC (MNI spatial coordinates: $x=-5, y=10, z=51$ ) significantly covaried with increases in response times for incongruent over neutral trials. Shown here and in subsequent figures are active clusters that surpassed a threshold of $z>2.3$ with cluster-based Gaussian random field correction.

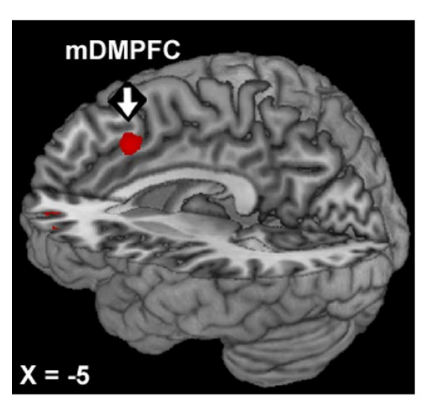

Decision Control

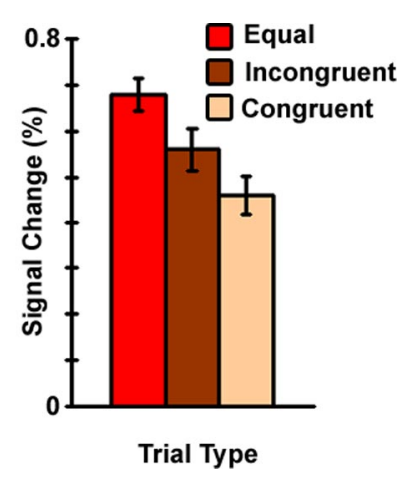

Figure 4. $\mathrm{mDMPFC}$ activation predicts decision-related control demands. Activation in the $\operatorname{DMPFC}(x=-6, y=24, z=38)$ during the choice phase of the decision-making task showed greater activation for both equal $>$ incongruent and incongruent $>$ congruent contrasts. Percentage signal change within this DMPFC cluster increased as the relative desirability of the decision options grew more similar, consistent with the attributed role of this cluster for indexing decision-related control. Error bars represent SEM.

related control demands, we found that subjects' response times were slowest for the equal trials, intermediate for incongruent trials, and fastest for congruent trials (supplemental Table S1, available at www.jneurosci.org as supplemental material). Finally, subjects also showed substantial individual variability in their choice preferences, providing a foundation for our analyses of strategic control (supplemental Fig. S1, available at www. jneurosci.org as supplemental material).

\section{Response-related control}

We found increased activation in the dorsomedial PFC, dorsolateral PFC, and parietal cortex for the incongruent condition in the Stroop task (supplemental Table S2, supplemental Fig. S2, available at www.jneurosci.org as supplemental material). When we used the difference in response time between incongruent and neutral conditions as a covariate, we found that activation in left dorsolateral prefrontal cortex and a posterior region of the DMPFC (pDMPFC) increased linearly with increasing response conflict (Fig. 3). The pDMPFC activation cluster was localized to the posterior part of the cingulate sulcus (BA 32). Activation within this cluster also correlated with an independent trait measure of motor impulsiveness (Patton et al., 1995) across subjects $(r=0.49, p<0.05)$ (supplemental Fig. S3, available at www. jneurosci.org as supplemental material).

\section{Decision-related control}

We next sought to identify brain regions whose activation was driven by the decision phase (when subjects deliberated between the two stock options) in the attribute-balancing task. We performed a conjunction analysis to identify regions showing significant effects for each of the two individual decision-related contrasts: equal $>$ incongruent and incongruent $>$ congruent. We found increased activation in right dorsolateral prefrontal cortex, right inferior parietal lobule, and a region of DMPFC anterior to that observed for response-related control (supplemental Fig. S4 A, supplemental Table S3, available at www.jneurosci.org as supplemental material). The DMPFC activation cluster for this contrast was also along the cingulate sulcus (BA 32), anterior to the cluster for response-related control, and we refer to it as $\mathrm{mDMPFC}$. Within this region, activation increased in a stepwise manner for our three levels of decision-related control (Fig. 4). Notably, these effects cannot be attributed to response conflict, given that they are time-locked to the decision phase that occurs before response mappings had been indicated to the subject.

\section{Strategy-related control}

Next, we sought to identify regions that indexed strategic control demands across subjects, using individuals' preference for the balancing strategy as a covariate in our between-subject analysis. (We report main-effect analyses unrelated to strategy-related control in the supplemental material; see supplemental Fig. S4 B, available at www.jneurosci.org as supplemental material.) We found that individual differences in strategic preferences across subjects predicted the relative activation evoked by balanced and extreme choices in only one region: anterior dorsomedial prefrontal cortex (aDMPFC) (Fig. 5), along the paracingulate region (BA 9). People who expressed a greater preference for the balanced option exhibited a greater increase in DMPFC activation for the extreme option compared to the balanced option and vice versa. The difference in activation also correlated with an independent trait measure of need for cognition (Epstein et al., 1996), which measures the relative cognitive effort associated with decision making $(r=0.60, p<0.005)$ (supplemental Fig. S5, available at www.jneurosci.org as supplemental material).

To verify that the clusters implicated in strategy- and decisionrelated control were functionally distinct, we conducted a post hoc regions of interest analysis within the aDMPFC and mDMPFC regions. We found that aDMPFC did not show a linear relationship with increasing decision conflict, nor did mDMPFC show a significant correlation with strategic variability across subjects (supplemental Fig. S6, available at www.jneurosci.org as supplemental material). Thus, we conclude that these regions made distinct functional contributions to performance of our experimental tasks.

\section{Evidence for a functional topography in DMPFC}

The results presented above provide strong evidence for a functional topography within the DMPFC: the most anterior cluster tracked strategy-related control, a middle cluster predicted decision-related control, and a more posterior cluster tracked response-related control (Fig. 6). To ascertain the consistency of these results with the previous literature, we conducted a meta- 
analysis of 53 studies that reported DMPFC activation in tasks involving decision making and/or cognitive control. We classified each study as involving either decision-related or response-related control, based on task properties, and included every reported coordinate of medial frontal cortex activation in an activation likelihood estimation analysis (see supplemental material, available at www.jneurosci.org). We exclude strategyrelated control in this meta-analysis due to lack of previous work in this area (but see De Martino et al., 2006; Venkatraman et al., 2009).

Our meta-analysis revealed a strong spatial dissociation within DMPFC between activations related to decisions and to responses, with more anterior regions being involved in decision making and more posterior regions with response-related cognitive control (supplemental Fig. S7, available at www.jneurosci.org as supplemental material). The centroids of maximum likelihood overlapped with the observed activations for decision-related and response-related control demands in our study (Fig. 6, solid squares), and both are posterior to the focus for strategy-related control in aDMPFC.

\section{Discussion}

We used tasks that evoked three distinct forms of control demands - response, decision, and strategy-and found strong evidence for a posterior to anterior topography within the DMPFC. Three distinct foci of activation were identified based on separate behavioral measures using data from the same individuals. These results both provide new insight into the functional organization of DMPFC and suggest a reconciliation of recent controversies about that region's role in complex decision-making and cognitive control.

Control demands play an important role in adaptive decision making, particularly since decision preferences are strongly influenced by context (Simonson and Tversky, 1992; Tversky and Simonson, 1993). For example, people avoid decision options that seem extreme, whether compared to other alternatives or whether having attributes with highly disparate values (Chernev, 2004,2005 ). Options that are balanced (e.g., their scores on various attributes are more equal) thus frequently serve as a desirable compromise relative to options containing attributes with high dispersion. We introduced a similar biasing context in the current study, which used an abstract market environment with anonymized options to control for subjects' differential prior exposure to stocks. This task allowed us to manipulate the degree of decision-related and strategy-related control independently, the former by varying the relative desirability of the choices and the latter by measuring individual variability in strategic bias.

Several aspects of our experimental design allow us to conclude that these distinct forms of control were represented in distinct regions within DMPFC. First, we explicitly separated the decision phase from the response phase so that activations related to decision control cannot be attributed to confounding effects from response selection or motor preparation (Pochon et al., 2008). Second, subjects received no immediate feedback about their decisions when choosing between different stocks; instead, feedback was provided in a separate, later run. This allowed us to rule out effects of outcome history when characterizing our subjects' decision preferences, while also precluding alternative explana-

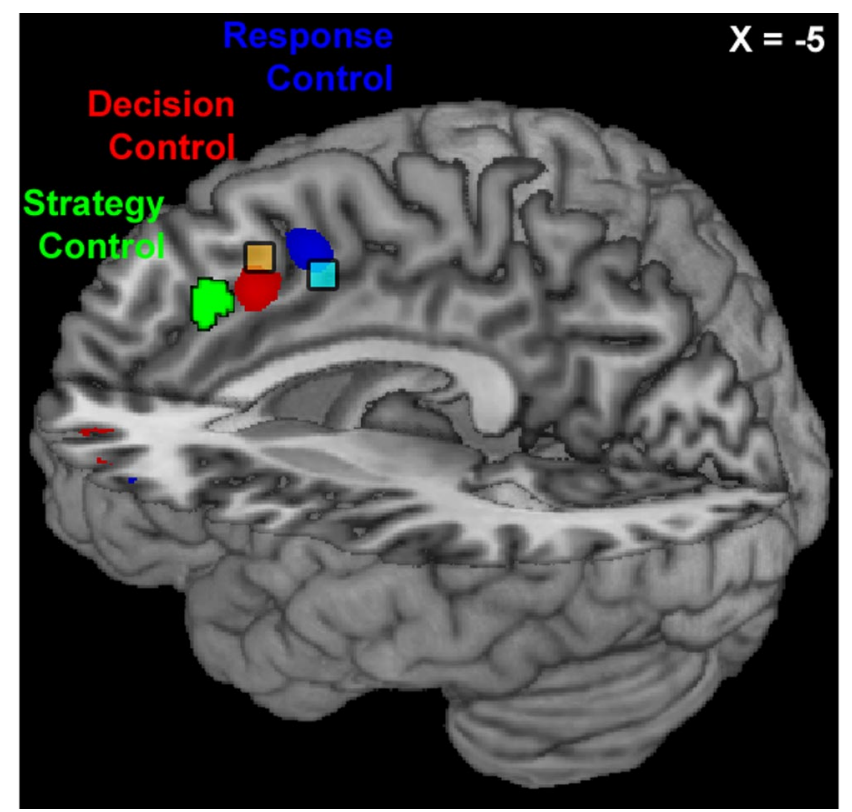

Figure 6. Summary of a functional topography within DMPFC. We found evidence for an anterior to posterior functional topography of the DMPFC with three distinct clusters predicting strategy (green), decision (red), and response (blue) control. We additionally conducted a meta-analysis that included studies of response-related control and decision making. Centroids of maximum activation likelihood for these two types of studies corresponded well to our postulated distinction within DMPFC, with the response-related control studies exhibiting activation in PDMPFC (blue square; $x=-6, y=6, z=42$ ) and the decision-making studies exhibiting activation in $\mathrm{mDMPFC}$ (red square; $x=-4, y=22, z=47$ ).

tions for the observed DMPFC activation like error detection, reinforcement learning, and signaling reward prediction errors (Kiehl et al., 2000; Kerns et al., 2004; Kennerley et al., 2006; Kim et al., 2006). We do not claim that DMPFC plays no role in these functions but note that they did not contribute to the activations observed in this experiment. Third, we used an independent counting Stroop task to elucidate neural mechanisms of response-related control. Fourth, all analyses were conducted within the same set of subjects, as critical for making clear spatial comparisons. And, finally, our distinct forms of control demands were each associated with a unique and independent behavioral covariate that was well controlled within each task, eliminating potential effects of taskspecific materials.

Several studies have found increased activation in the DMPFC associated with complex decision making (Paulus et al., 2002; 
Walton et al., 2003; Rushworth et al., 2004; Zysset et al., 2006; Pochon et al., 2008; Botvinick and Rosen, 2009), though these findings could be confounded by activation related to response preparation (Pochon et al., 2008). A more recent study using a perceptual decision-making task sought to explore the role of DMPFC in decision conflict, defined as the difficulty arising from choosing between two equally likely choices, while accounting for response-related activation (Pochon et al., 2008). They demonstrated greater DMPFC activation for decisions involving alternatives that were equally attractive, even in the absence of an explicit response (e.g., precluding an explanation in terms of response-related control). Activation within this region also varied with subjective ratings of decision difficulty. While Pochon et al. did not include additional conditions to allow testing of topographic organization, the manipulation of decision conflict in that study led to activation in regions of the DMPFC similar to those associated with decision-related control in our current study.

Moreover, there are initial suggestions that DMPFC is also recruited when individuals make choices that run counter to general behavioral tendencies or strategies (Paulus et al., 2002; De Martino et al., 2006; Hampton and O'Doherty, 2007), although these have heretofore been discussed in terms of decision preferences. Studies involving complex decision making have often focused on identifying brain systems that shape behavior toward or against particular choices. Yet, it is becoming increasingly apparent that people employ a variety of strategies to simplify the representations of decision problems and reduce computational demands (Tversky and Kahneman, 1974; Payne et al., 1988, 1992; Gigerenzer and Goldstein, 1996). In this study, we show that activation in a similar region of the DMPFC predicts variability in strategy-related control across subjects. Specifically, individuals with greater bias for the balancing strategy exhibited a greater increase in activation for the extreme choices compared to the balanced choices and vice versa. Strikingly, the cluster within DMPFC associated with strategy-related control was anterior to and functionally distinct from the clusters associated with decision-related control and response-related control. Therefore, a parsimonious explanation for the role of DMPFC in complex decision making is that distinct functional clusters might be associated with distinct aspects of decision making, including strategy preferences and response preparation.

One potential conjecture for functional specialization within the DMPFC is related to differences in connectivity of these clusters to other regions in the brain. A commonly held framework, one advanced in different guises by different theorists, suggests that lateral prefrontal cortex contains a topographic organization along its posterior to anterior axis (Koechlin et al., 2000, 2003). More posterior regions, those immediately adjacent to premotor cortex, are associated with setting up general rules for behavior. Conversely, more anterior regions support the instantiation of rules for behavior based on the current context. Findings from functional neuroimaging studies argue for further divisions within anterior prefrontal cortex, such that regions around the frontal pole support relational integration, or the combination of disparate information into a single judgment (Christoff et al., 2001). We speculate that the different regions of DMPFC differ in their lateral prefrontal targets.

Initial evidence for such a functional organization stems from a recent study that demonstrates choice-specific changes in the functional connectivity of the DMPFC with other regions involved in decision making (Venkatraman et al., 2009). Similarly, Beckmann et al. (2009) used magnetic resonance diffusion tractography to delineate probabilistic anatomical connectivity of the cingulate cortex to other brain regions. The authors found that the lateral prefrontal cortex exhibited the highest probability of anatomical connectivity with a cluster that corresponds spatially to the region that predicts decision-related control in our study, while the premotor and precentral cortices showed highest probability of connection with a cluster that predicted responserelated control in the present study. Under this perspective, the current results point to a generalized role for the DMPFC in cognitive control, but specific computational roles for its subregions depending upon the task demands and current context. Elucidating the functional connectivity of the different clusters in the DMPFC with other brain regions-ideally, using within-task measures derived from the same subjects-may hold the key to fully understanding its role in decision making.

\section{References}

Amodio DM, Frith CD (2006) Meeting of minds: the medial frontal cortex and social cognition. Nat Rev Neurosci 7:268-277.

Beckmann M, Johansen-Berg H, Rushworth MF (2009) Connectivity-based parcellation of human cingulate cortex and its relation to functional specialization. J Neurosci 29:1175-1190.

Behrens TE, Hunt LT, Woolrich MW, Rushworth MF (2008) Associative learning of social value. Nature 456:245-249.

Behrens TE, Hunt LT, Rushworth MF (2009) The computation of social behavior. Science 324:1160-1164.

Botvinick MM, Rosen ZB (2009) Anticipation of cognitive demand during decision-making. Psychol Res 73:835-842.

Botvinick MM, Braver TS, Barch DM, Carter CS, Cohen JD (2001) Conflict monitoring and cognitive control. Psychol Rev 108:624-652.

Brainard DH (1997) The psychophysics toolbox. Spat Vis 10:433-436.

Bush G, Whalen PJ, Rosen BR, Jenike MA, McInerney SC, Rauch SL (1998) The counting Stroop: an interference task specialized for functional neuroimaging-validation study with functional MRI. Hum Brain Mapp 6:270-282.

Bush G, Luu P, Posner MI (2000) Cognitive and emotional influences in anterior cingulate cortex. Trends Cogn Sci 4:215-222.

Bush G, Vogt BA, Holmes J, Dale AM, Greve D, Jenike MA, Rosen BR (2002) Dorsal anterior cingulate cortex: a role in reward-based decision making. Proc Natl Acad Sci U S A 99:523-528.

Carter CS, Braver TS, Barch DM, Botvinick MM, Noll D, Cohen JD (1998) Anterior cingulate cortex, error detection, and the online monitoring of performance. Science 280:747-749.

Chernev A (2004) Extremeness aversion and attribute-balance effects in choice. J Consum Res 31:259-263.

Chernev A (2005) Context effects without a context: attribute balance as a reason for choice. J Consum Res 32:213-223.

Christoff K, Prabhakaran V, Dorfman J, Zhao Z, Kroger JK, Holyoak KJ, Gabrieli JD (2001) Rostrolateral prefrontal cortex involvement in relational integration during reasoning. Neuroimage 14:1136-1149.

Christoff K, Ream JM, Geddes LP, Gabrieli JD (2003) Evaluating selfgenerated information: anterior prefrontal contributions to human cognition. Behav Neurosci 117:1161-1168.

De Martino B, Kumaran D, Seymour B, Dolan RJ (2006) Frames, biases, and rational decision-making in the human brain. Science 313:684-687.

Epstein S, Pacini R, Denes-Raj V, Heier H (1996) Individual differences in intuitive-experiential and analytical-rational thinking styles. J Pers Soc Psychol 71:390-405.

Etkin A, Egner T, Peraza DM, Kandel ER, Hirsch J (2006) Resolving emotional conflict: a role for the rostral anterior cingulate cortex in modulating activity in the amygdala. Neuron 51:871-882.

Gehring WJ, Willoughby AR (2002) The medial frontal cortex and the rapid processing of monetary gains and losses. Science 295:2279-2282.

Gigerenzer G, Goldstein DG (1996) Reasoning the fast and frugal way: models of bounded rationality. Psychol Rev 103:650-669.

Hampton AN, O'Doherty JP (2007) Decoding the neural substrates of reward-related decision making with functional MRI. Proc Natl Acad Sci U S A 104:1377-1382.

Kennerley SW, Walton ME, Behrens TE, Buckley MJ, Rushworth MF (2006) Optimal decision making and the anterior cingulate cortex. Nat Neurosci 9:940-947. 
Kerns JG, Cohen JD, MacDonald AW 3rd, Cho RY, Stenger VA, Carter CS (2004) Anterior cingulate conflict monitoring and adjustments in control. Science 303:1023-1026.

Kiehl KA, Liddle PF, Hopfinger JB (2000) Error processing and the rostral anterior cingulate: an event-related fMRI study. Psychophysiology $37: 216-223$.

Kim H, Shimojo S, O’Doherty JP (2006) Is avoiding an aversive outcome rewarding? Neural substrates of avoidance learning in the human brain. PLoS biology 4:e233.

Koechlin E, Corrado G, Pietrini P, Grafman J (2000) Dissociating the role of the medial and lateral anterior prefrontal cortex in human planning. Proc Natl Acad Sci U S A 97:7651-7656.

Koechlin E, Ody C, Kouneiher F (2003) The architecture of cognitive control in the human prefrontal cortex. Science 302:1181-1185.

Mériau K, Wartenburger I, Kazzer P, Prehn K, Lammers CH, van der Meer E, Villringer A, Heekeren HR (2006) A neural network reflecting individual differences in cognitive processing of emotions during perceptual decision making. Neuroimage 33:1016-1027.

Patton JH, Stanford MS, Barratt ES (1995) Factor structure of the Barratt impulsiveness scale. J Clin Psychol 51:768-774.

Paulus MP, Hozack N, Frank L, Brown GG (2002) Error rate and outcome predictability affect neural activation in prefrontal cortex and anterior cingulate during decision-making. Neuroimage 15:836-846.

Payne JW, Bettman JR, Johnson EJ (1988) Adaptive strategy selection in decision-making. J Exp Psychol Learn Mem Cogn 14:534-552.

Payne JW, Bettman JR, Coupey E, Johnson EJ (1992) A constructive process view of decision-making - multiple strategies in judgment and choice. Acta Psychologica 80:107-141.

Pelli DG (1997) The VideoToolbox software for visual psychophysics: transforming numbers into movies. Spat Vis 10:437-442.

Pochon JB, Riis J, Sanfey AG, Nystrom LE, Cohen JD (2008) Functional imaging of decision conflict. J Neurosci 28:3468-3473.
Ridderinkhof KR, Ullsperger M, Crone EA, Nieuwenhuis S (2004) The role of the medial frontal cortex in cognitive control. Science 306:443-447.

Rorden C, Karnath HO, Bonilha L (2007) Improving lesion-symptom mapping. J Cogn Neurosci 19:1081-1088.

Rushworth MF, Behrens TE (2008) Choice, uncertainty and value in prefrontal and cingulate cortex. Nat Neurosci 11:389-397.

Rushworth MF, Walton ME, Kennerley SW, Bannerman DM (2004) Action sets and decisions in the medial frontal cortex. Trends Cogn Sci 8:410-417.

Rushworth MF, Kennerley SW, Walton ME (2005) Cognitive neuroscience: resolving conflict in and over the medial frontal cortex. Curr Biol 15:R54-R56.

Simonson I, Tversky A (1992) Choice in context: tradeoff contrast and extremeness aversion. J Market Res 29:281-295.

Smith SM, Jenkinson M, Woolrich MW, Beckmann CF, Behrens TE, Johansen-Berg H, Bannister PR, De Luca M, Drobnjak I, Flitney DE, Niazy RK, Saunders J, Vickers J, Zhang Y, De Stefano N, Brady JM, Matthews PM (2004) Advances in functional and structural MR image analysis and implementation as FSL. Neuroimage 23 [Suppl 1]:S208-S219.

Tversky A, Kahneman D (1974) Judgment under uncertainty: heuristics and biases. Science 185:1124-1131.

Tversky A, Simonson I (1993) Context-dependent preferences. Manage Sci 39:1179-1189.

Venkatraman V, Payne JW, Bettman JR, Luce MF, Huettel SA (2009) Separate neural mechanisms underlie choices and strategic preferences in risky decision making. Neuron 62:593-602.

Walton ME, Bannerman DM, Alterescu K, Rushworth MF (2003) Functional specialization within medial frontal cortex of the anterior cingulate for evaluating effort-related decisions. J Neurosci 23:6475-6479.

Zysset S, Wendt CS, Volz KG, Neumann J, Huber O, von Cramon DY (2006) The neural implementation of multi-attribute decision making: a parametric fMRI study with human subjects. Neuroimage 31:1380-1388. 